\title{
Rutura Crónica Bilateral do Tendão Quadricipital: Diagnóstico Tardio e Reparação Direta
}

\section{Bilateral Quadriceps Tendon Rupture: Late Diagnosis and Direct Repair}

Rita Sapage ${ }^{1,3}$, Pedro Teixeira da Mota ${ }^{1,3}$, Carlos Branco ${ }^{1,3}$, Rita Sousa ${ }^{1,3}$, Diogo Sousa ${ }^{1,3}$, Carlos Pintado 2,3

Autor Correspondente/Corresponding Author:

Rita Sapage [rita.sapage@hotmail.com] Av. Noruega, Lordelo, 5000-508 Vila Real, Portugal ORCID iD: 0000-0003-4720-9656

\section{RESUMO}

A rutura bilateral do tendão quadricipital é uma lesão rara, não sendo muitas vezes diagnosticada na apresentação inicial.

Apresenta-se o caso de um doente de 41 anos, género masculino, sem antecedentes médico-cirúrgicos ou medicação habitual, com diagnóstico tardio de rutura bilateral do tendão quadricipital (3 meses de evolução) após traumatismo de baixa intensidade. Foi submetido a reparação do tendão quadricipital com sutura transrotuliana e ancoragens à rótula bilateralmente. O pós-operatório decorreu sem intercorrências, tendo iniciado programa de reabilitação funcional com obtenção de arco de mobilidade de 0-120 aos 6 meses de seguimento.

O diagnóstico de rutura bilateral do tendão quadricipital requer elevado grau de suspeição clínica pela sua raridade e variabilidade de apresentação. O tratamento precoce poderá estar associado a menor dificuldade cirúrgica e melhor resultado funcional. No presente caso foi possível a reparação direta com sutura transrotuliana do tendão e reforço de âncoras, com recuperação funcional sobreponível ao estado prévio ao traumatismo.

PALAVRAS-CHAVE: Lesões do Joelho; Lesões dos Tendões; Músculo Quadríceps/lesão; Rutura Espontânea

1.Interno de Formação Específica em Ortopedia e Traumatologia, Serviço de Ortopedia e Traumatologia, Centro Hospitalar de Trás-os-Montes e Alto Douro, Vila Real, Portugal. 2. Diretor de Serviço de Ortopedia e Traumatologia, Serviço de Ortopedia e Traumatologia, Centro Hospitalar de Trás-os-Montes e Alto Douro, Vila Real, Portugal. 3. Serviço de Ortopedia e Traumatologia, Centro Hospitalar de Trás-os-Montes e Alto Douro, Vila Real, Portugal. 


\begin{abstract}
Bilateral quadriceps tendon rupture is a rare lesion and is often missed at initial evaluation.

We describe the case of a 41 -year-old male, with no past medical history or current medications, late diagnosed with bilateral quadriceps tendon rupture ( 3 months of evolution) after minor trauma. He was submitted to bilateral tendon repair with transpatellar fixation and anchor suture. Satisfactory post-operative outcomes were achieved following rehabilitative physiotherapy. At 6 months follow-up passive and active range of movement 0 to $120^{\circ}$ could be achieved.

For its rarity and variable clinical presentation, bilateral quadriceps tendon rupture diagnosis requires a high level of clinical suspicion. Early treatment has been associated to less demanding surgical technique and better functional outcome. In the present case it was possible to achieve direct repair with transpatellar fixation and anchor suture, with functional recovery to previous healthy state.
\end{abstract}

KEYWORDS: Knee Injuries; Quadriceps Muscle/injuries; Rupture, Spontaneous; Tendon Injuries

\section{INTRODUÇÃO}

A rutura bilateral do tendão quadricipital é uma lesão rara, sendo cerca de quinze a vinte vezes menos frequente do que a rutura unilateral. A maioria das lesões bilaterais ocorre simultaneamente. ${ }^{1}$ A rutura localiza-se geralmente na junção osteotendinosa e na presença de alterações tendinosas degenerativas. ${ }^{2}$ Ocorre mais frequentemente em homens, com idade superior a 50 anos. A etiologia pode ser traumática ou espontânea. ${ }^{3}$ Até $85 \%$ dos doentes, pode ter uma condição médica associada que predispõe a rutura. ${ }^{1,2}$ Pode estar associada a fatores de risco como obesidade, doença renal crónica, hiperparatiroidismo, doenças reumatoides, uso de esteroides e injeções intra-articulares. Nestes casos, estão descritas ruturas espontâneas sem traumatismo evidente. ${ }^{4}$ As ruturas bilaterais ocorrem após traumatismo apenas em cerca de $30 \%$ dos casos. ${ }^{3}$

O diagnóstico é essencialmente clínico, complementado pelas técnicas de imagem. Pode ser referida história de dor prévia que poderá traduzir patologia tendinosa subjacente. Clinicamente deve ser procurada dor local, defeito palpável cerca de $2 \mathrm{~cm}$ acima do polo superior da rótula, incapacidade de realizar extensão da perna contra resistência e de elevação do membro inferior em extensão, neste caso em ruturas completas do tendão quadricipital. Devem ser realizadas radiografias de face e de perfil, com evidência de patela baja, no entanto raramente são suficientes para confirmação diagnóstica. ${ }^{5}$ A ecografia e a ressonância magnética estão indicadas em casos de incerteza diagnóstica e na distinção entre ruturas completas ou parciais., $\mathrm{A}$ ecografia pode ser útil no diagnóstico precoce e apresenta elevada acuidade na avaliação tendinosa. ${ }^{7}$ Embora possa ser considerada o método de imagem de eleição, a ressonância magnética é um exame mais caro e muitas vezes não se encontra disponível em contexto de urgência. Em alguns casos, especialmente em doentes mais jovens e em traumatismos de maior intensidade, providencia informação adicional sobre lesões intra-articulares associadas como lesões ligamentares e meniscais. ${ }^{5}$

O tratamento cirúrgico simultâneo é o método de tratamento preferencial. Ruturas do tendão quadricipital com mais de duas semanas podem retrair até cinco centímetros. Em ruturas crónicas, para além da reparação direta utilizada em situações agudas, pode ser necessário recorrer a técnicas de alongamento tendinoso (como a operação de Codivilla de alongamento $\mathrm{V}$-Y) ou ao uso de auto ou alo-enxerto. ${ }^{6,8}$

Em termos de prognóstico funcional, uma percentagem significativa dos doentes apresenta dor residual, perda de força muscular quadricipital e incapacidade funcional (até 50\% dos doentes são incapazes de retornar ao nível de atividade física prévia). ${ }^{2}$

\section{CASO CLÍNICO}

Os autores descrevem o caso clínico de um homem de 41 anos, sem antecedentes médico-cirúrgicos conhecidos e sem medicação habitual, com índice de massa corporal de 24,5 e com atividade desportiva regular (futebol amador). Foi observado no Serviço de Urgência por traumatismo de ambos os joelhos após queda em velocidade durante jogo de futebol, tendo realizado radiografia de face e perfil dos joelhos sem diagnóstico de lesão aparente. Foi prescrita medicação analgésica para controlo da dor e o doente manteve inicialmente capacidade de marcha com apoio bilateral de canadianas. Por persistência de gonalgia bilateral e incapacidade progressiva de marcha, foi novamente observado após 3 meses do traumatismo inicial. Clinicamente apresen- 
tava bilateralmente dor ao nível do polo superior da rótula, depressão supra-rotuliana palpável e incapacidade de extensão contra resistência de ambas as pernas. Nas radiografias de face e perfil dos joelhos apresentava desvio inferior de ambas as rótulas, sem aparente evidência de fratura (Fig. 1). Realizou ressonância magnética que demonstrou rutura completa bilateral do tendão quadricipital (Fig. 2). Intra-operatoriamente, após lise de aderências peri-tendinosas e musculares, foi obtida mobilidade suficiente de forma a realizar reparação tendinosa direta com tenorrafia topo-a-topo. Não se verificou atrofia muscular significativa o que poderá estar associado ao nível de atividade desportiva do doente e poderá justificar a menor retração do tendão. $\bigcirc$ doente foi, então, submetido a reparação do tendão quadricipital com sutura tipo Krakow, tunelizações transrotulianas e ancoragens à rótula bilateralmente (âncoras 5,5 mm com fio de sutura não-absorvível) (Fig.s 3 e 4). 0 pós-operatório imediato decorreu sem intercorrências. Após 9 dias, teve alta hospitalar para o domicílio com indicação para marcha com canadianas que cumpriu durante 4 semanas. Manteve tala imobilizadora DePuy em $0^{\circ}$ de extensão durante 8 semanas. Iniciou posteriormente programa de reabilitação funcional, que incluiu inicialmente fortalecimento muscular isométrico e mobilização ativa assistida, com progressivo fortalecimento isotónico concêntrico. Na consulta de 3 meses pós-operatório, apresenta-se sem dor, a deambular sem apoio externo, com arco de mobilidade de ambos os joelhos de aproximadamente $0-120^{\circ}$, com ganho de massa e força muscular (grau 4/5) e com grau de satisfação elevado

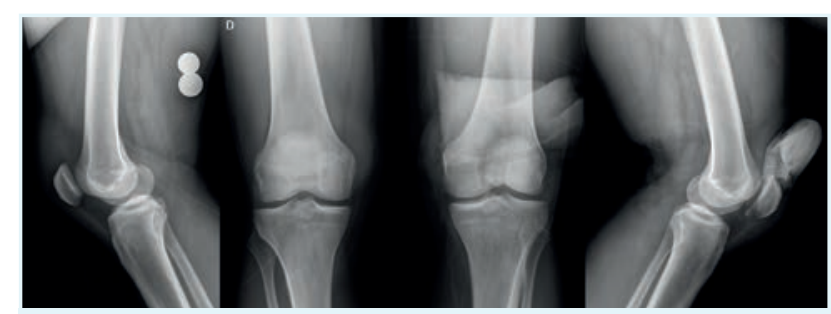

FIGURA 1. Radiografia de face e perfil do joelho direito e esquerdo.

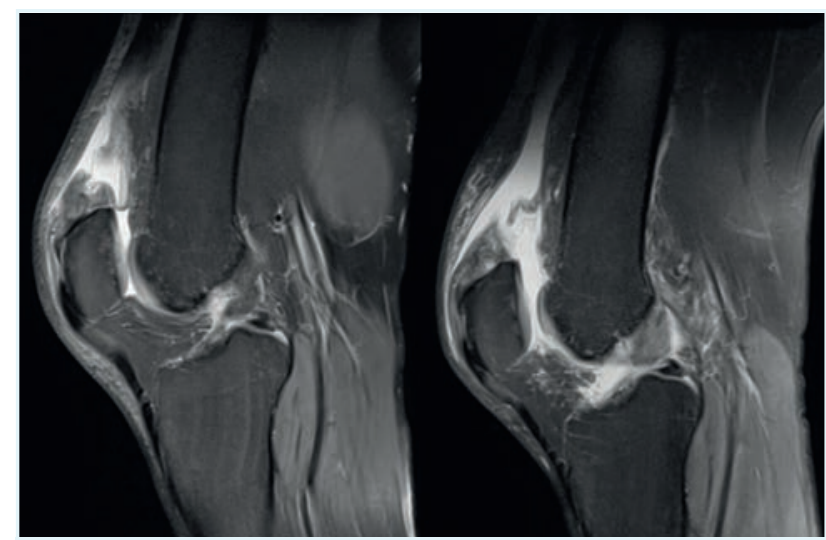

FIGURA 2. Ressonância magnética do joelho direito e esquerdo. (nega limitação funcional importante das atividades de vida diárias). Com 6 meses de seguimento apresenta-se clinicamente sobreponível, com recuperação de força muscular ao nível prévio ao traumatismo (Fig. 5).

\section{DISCUSSÃO}

A rutura bilateral do tendão quadricipital tem sido descrita apenas sob a forma de caso clínico ou como pequenas séries de casos. ${ }^{2}$ Também a variabilidade na apresentação clínica pode acrescer na dificuldade diagnóstica. As ruturas espontâneas bilaterais do aparelho extensor são duas vezes mais frequentes do que as traumáticas. ${ }^{3}$ As primeiras estão geralmente associadas a condições médicas predisponentes. $\bigcirc$ mecanismo traumático comummente descrito é a contração reflexa excêntrica súbita do músculo quadricipital, com o pé ancorado no solo e o joelho fletido. ${ }^{9}$ No caso apresentado não há conhecimento de nenhuma patologia médica prévia, tendo a rutura ocorrido após evento traumático. Está, por isso, englobado numa pequena minoria de doentes descritos na literatura. 2,10 Desta forma, é necessário um elevado grau de suspeição clínica para o reconhecimento destas lesões. ${ }^{3}$ A presença de esporões no polo proximal da rótula no local de inserção do tendão quadricipital também tem sido sugerida como fator de risco associado a rutura, o que poderá constituir um sinal de alerta na avaliação radiográfica destes doentes. ${ }^{9}$

Por serem entidades raras e de difícil diagnóstico, ruturas bilaterais simultâneas podem não ser diagnosticadas no momento de apresentação em 50\% dos casos. Está descrito como sendo comum um atraso de diagnóstico em média de 65 dias, o que poderá ter repercussões no resultado cirúrgico e funcional dado serem lesões graves e incapacitantes. $3,6,11$

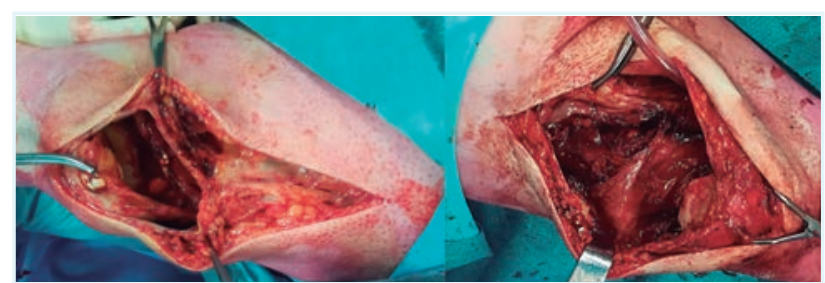

FIGURA 3. Fotografia intra-operatória de rutura do tendão quadricipital esquerdo e direito.

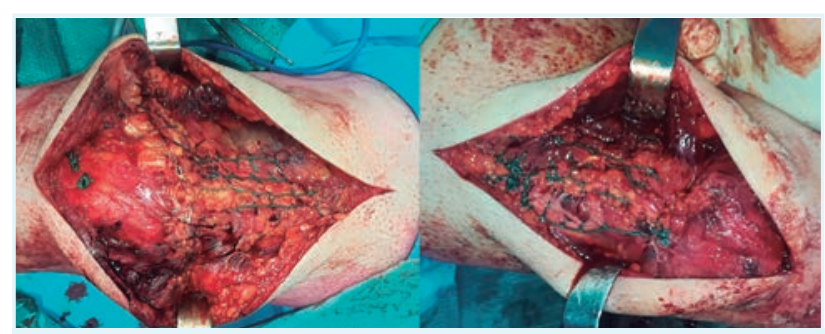

FIGURA 4. Reparação cirúrgica direta do tendão quadricipital esquerdo e direito com sutura transrotuliana e ancoragens à rótula. 


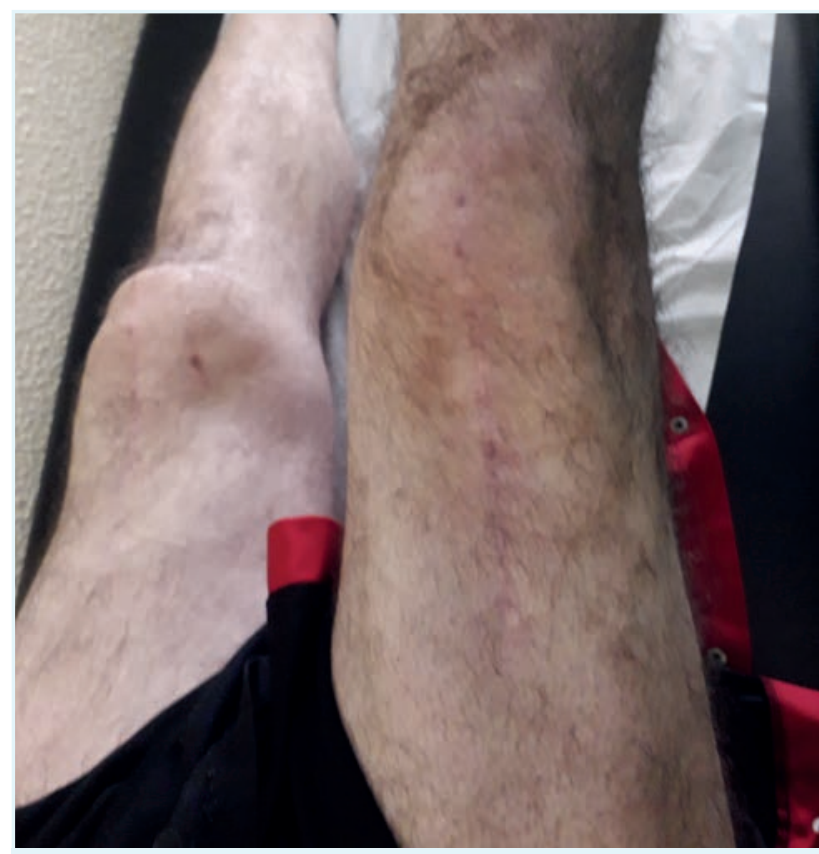

FIGURA 5. Pós-operatório ao sexto mês.

O tratamento cirúrgico pode ser desafiante. A literatura descrita no caso de ruturas crónicas do tendão quadricipital é igualmente escassa. Estes procedimentos são acrescidos de dificuldade pelo maior defeito tendinoso e pela atrofia muscular associada. Poderão estar associadas a retração do tendão superior a $5 \mathrm{~cm} .{ }^{1}$ Estão descritas várias técnicas cirúrgicas, como a reparação do tendão com sutura transrotuliana, a operação de Codivilla (com tenoplastia de alongamento) e a reconstrução com enxerto autólogo. Alguns autores descrevem não haver diferença no resultado funcional alcançado com os diversos procedimentos ou períodos subsequentes de imobilização. 2,8 No caso apresentado, foi possível a aproximação direta e a sutura transrotuliana do tendão com reforço de âncoras. Levanta-se a possibilidade de o grau de atrofia muscular constatada intra-operatoriamente ser menor do que o esperado no caso de uma rutura crónica, dado o nível habitual de atividade desportiva do doente e o facto de ter mantido a capacidade de marcha até à cirurgia.

A rutura bilateral crónica do tendão quadricipital é descrita como sendo de difícil tratamento e de longo período de recuperação. ${ }^{12} \mathrm{O}$ seu tratamento deve ser seguido de reabilitação funcional intensiva. ${ }^{2} \mathrm{O}$ diagnóstico e tratamento precoces são habitualmente descritos em associação a melhor resultado funcional. ${ }^{11,13} \mathrm{Um}$ atraso de tratamento de mais de duas semanas pode comprometer a reparação tendinosa primária pela sua retração. ${ }^{13}$ No entanto, há autores que descrevem apenas uma pequena percentagem de re-rutura do tendão previamente operado, sem relação com o atraso de diagnóstico e tratamento cirúrgico. ${ }^{3}$ Alguns autores não encontraram diferença no resultado funcional resultante de diferentes tempos de espera cirúrgica ${ }^{2}$ ou quando em comparação com ruturas unilaterais. ${ }^{11}$ No caso apresentado, o resultado pós-operatório foi globalmente satisfatório (com recuperação funcional sobreponível ao estado prévio ao traumatismo) apesar de a cirurgia ter sido realizada tardiamente.

Dada a escassez de literatura disponível sobre este tema, especialmente sobre ruturas bilaterais, ${ }^{3,11}$ reveste-se de importância a exposição do caso clínico apresentado.

CONFLITOS DE INTERESSE: Os autores declaram não ter qualquer conflito de interesse na realização do presente trabalho.

FONTES DE FINANCIAMENTO: Não houve qualquer fonte de financiamento na realização do presente trabalho.

CONFIDENCIALIDADE DOS DADOS: Os autores declaram ter seguido os protocolos da sua instituição acerca da publicação dos dados de doentes.

CONSENTIMENTO: Consentimento do doente para publicação obtido.

PROVENIÊNCIA E REVISÃO POR PARES: Não comissionado; revisão externa por pares.

CONFLICTS OF INTEREST: The authors declare that they have no conflicts of interest.

FINANCIAL SUPPORT: This work has not received any contribution, grant or scholarship.

CONFIDENTIALITY OF DATA: The authors declare that they have followed the protocols of their work center on the publication of data from patients.

PATIENT CONSENT: Consent for publication was obtained.

PROVENANCE AND PEER REVIEW: Not commissioned; externally peer reviewed.

\section{REFERÊNCIAS}

1. Rehman H, Kovacs P. Quadriceps tendon repair using hamstring, prolene mesh and autologous conditioned plasma augmentation. A novel technique for repair of chronic quadriceps tendon rupture. Knee. 2015;22):664-8. doi: 10.1016/j. knee.2015.04.006.

2. Moura DL, Marques JP, Pinheiro JP, Fonseca F. Total bilateral ruptures of the knee extensor apparatus. Rev Bras Ortop. 2017;52:663-9. doi: 10.1016/j.rboe.2016.11.009.

3. Camarda L, D’Arienzo A, Morello S, Guarneri M, Balistreri F, D'Arienzo M. Bilateral ruptures of the extensor mechanism of the knee: A systematic review. J Orthop. 2017;14:445-53. doi: 10.1016/j.jor.2017.07.008. 
4. Artan AS, Basgoze B. Bilateral quadriceps tendon rupture in a hemodialysis patient. Clin Exp Nephrol. 2015;19:755-6. doi: 10.1007/s10157-015-1089-z.

5. Nelson M, Jersey A, Okumura Y, Stankard B. Bedside ultrasound diagnosis of quadriceps tendon rupture and avulsed patella. Curr Sports Med Rep. 2017;16:153-5. doi: 10.1249/ JSR.0000000000000354.

6. Neubauer T, Wagner M, Potschka T, Riedl M. Bilateral, simultaneous rupture of the quadriceps tendon: a diagnostic pitfall? Report of three cases and meta-analysis of the literature. Knee Surg Sports Traumatol Arthrosc. 2007;15:43-53

7. Prado-Costa R, Rebelo J, Monteiro-Barroso J, Preto AS. Ultrasound elastography: compression elastography and shear-wave elastography in the assessment of tendon injury. Insights Imaging. 2018;9:791-814. doi: 10.1007/s13244-018-06421.

8. Forslund J, Gold S, Gelber J. Allograft reconstruction of a chronic quadriceps tendon rupture with use of a novel technique. JBJS Case Connect. 2014;4:e42-e2. doi: 10.2106/JBJS. CC.M.00230.

9. Pagliari M, Menna CR, Migliorini A, Molinari M. Atraumatic acute bilateral quadriceps tendon rupture in a patient with bilateral patella spurs. A case report and review of literature. Acta Biomed. 2018;90:203-8.

10. Gaheer RS, Hawkins A. Rapid recovery from spontaneous and simultaneous bilateral quadriceps tendon rupture in an active, healthy individual. Orthopedics. 2010;33:512. doi: 10.3928/01477447-20100526-22.

11. Chang ES, Dodson CC, Tjoumakaris F, Cohen SB, Chang ES, Dodson CC, et al. Functional results following surgical repair of simultaneous bilateral quadriceps tendon ruptures. Phys Sportsmed. 2014;42:114-8. doi: 10.3810/psm.2014.05.2063.

12. Seifert C, Vokes J, Roberts A, Gorczyca J, Judd K. Simultaneous bilateral extensor mechanism disruptions: more than double the trouble? J Knee Surg. 2019 (in press). doi: 10.1055/ s-0039-1688779.

13. Siwek CW, Rao JP. Ruptures of the extensor mechanism of the knee joint. J Bone Joint Surg Am. 1981;63:932-7. 